\title{
On the Normality of Arithmetical Constants
}

\author{
Jeffrey C. Lagarias
}

\section{CONTENTS}

1. Introduction

2. Radix Expansions

3. Perturbed Radix Expansions

4. BBP-Numbers and Hypothesis A

5. Special Values of G-Functions

6. Invariant Measures and Furstenberg's Conjecture

7. Concluding Remarks

Acknowledgements

References

2000 AMS Subject Classification: Primary, 11K16; Secondary, 11A63, 28D05, 37E05

Keywords: dynamical systems, invariant measures, $G$-functions, polylogarithms, radix expansions
Bailey and Crandall recently formulated "Hypothesis A", a general principle to explain the (conjectured) normality of the binary expansion of constants like $\pi$ and other related numbers, or more generally the base b expansion of such constants for an integer $b \geq 2$. This paper shows that a basic mechanism underlying their principle, which is a relation between single orbits of two discrete dynamical systems, holds for a very general class of representations of numbers. This general class includes numbers for which the conclusion of Hypothesis $A$ is not true. The paper also relates the particular class of arithmetical constants treated by Bailey and Crandall to special values of G-functions, and points out an analogy of Hypothesis A with Furstenberg's conjecture on invariant measures.

\section{INTRODUCTION}

Much is known about the irrationality and transcendence of classical arithmetical constants such as $\pi$, $e$, and $\zeta(n)$ for $n \geq 2$. There are general methods which in many cases establish irrationality or transcendence of such numbers. In contrast, almost nothing is known concerning the question of whether arithmetical constants are normal numbers to a fixed base, say $b=2$. It is unknown whether any algebraic number is normal to any integer base $b \geq 2$. Even very weak assertions in the direction of normality are unresolved. For example, it is not known whether arbitrarily long blocks of zeros appear in the binary expansion of $\sqrt{2}$.

Bailey and Crandall [2001] formulated what they called "Hypothesis A", which provides a hypothetical general principle to explain the (conjectured) normality to base 2 of certain arithmetical constants such as $\pi$ and $\log 2$.

Hypothesis A. Consider a positive integer $b \geq 2$ and a rational function $R(x)=p(x) / q(x) \in \mathbb{Q}(x)$ such that $\operatorname{deg} p(x)<\operatorname{deg} q(x)$ and that $q(x)$ has no nonnegative integer roots. Let $\theta=\sum_{n=0}^{\infty} p(n) / q(n) b^{-n}$, 
and define a sequence $\left\{y_{n}: n \geq 0\right\}$ by setting $y_{0}=0$,

$$
y_{n+1}=b y_{n}+\frac{p(n)}{q(n)}(\bmod 1) .
$$

Then this sequence either has finitely many limit points or is uniformly distributed mod 1.

This hypothesis concerns the behavior of a particular orbit of the discrete dynamical system (1-1). Assuming Hypothesis A, Bailey and Crandall deduced that the number $\theta$ either is rational or else is a normal number to base $b$; these correspond to the two possible behaviors of the sequence $\left\{y_{n}: n \geq 1\right\}$ allowed by Hypothesis A, see Theorem 4.2 below. Proving Hypothesis A appears intractable, but it seems useful in collecting a number of disparate phenomona together under a single principle. A formulation in terms of dynamical systems is natural, because the property of normality is itself expressable in terms of dynamics of an orbit of another dynamical system, the $b$-transformation, see Section 2 . The basic mechanism rendering Hypothesis A useful is a relation between particular orbits of these two different dynamical systems.

This paper provides some complements to the results of Bailey and Crandall. It shows that the relation between particular orbits of two discrete dynamical systems underlying Hypothesis $A$ is valid very generally, in that it applies to expansions of real numbers of the form, $\theta=\sum_{n=1}^{\infty} \varepsilon_{n} b^{-n}$, with $\varepsilon_{n}$ arbitrary real numbers with $\varepsilon_{n} \rightarrow 0$ as $n \rightarrow \infty$; see Theorem 3.1. Every real number has such an expansion. Hypothesis A is not true in such generality, so in order to be valid Hypothesis A must be restricted to apply only to expansions of some special form. Bailey and Crandall do this, formulating Hypothesis A only for a countable class of arithmetical constants which in the sequel we call $B B P$-numbers. It does not seem clear what should be the "optimal" class of arithmetical constants for which Hypothesis A might be valid. The remainder of the paper discusses various mathematical topics relevant to this issue. We relate BBP-numbers to the theory of $G$-functions and characterize the subclass of BBP-numbers which are "special values" of $G$-functions. We also compare Hypothesis A to a conjecture of Furstenberg in ergodic theory, and this suggests some further questions to pursue.
We now summarize the contents of the paper in more detail. In Sections 2 and 3 we give the dynamical connection underlying Hypothesis A. In Section 2 we review radix expansions to an integer base $b \geq 2$ treated as a discrete dynamical system acting on the interval $[0,1]$. The radix expansion of a real number $\theta$ is described by an orbit of a dynamical system, the $b$-transformation $T_{b}(x)=b x(\bmod 1)$, studied by Rényi [1957] and Parry [1960]. For a given number $\theta$ its $b$-expansion can be computed from the iterates of this system

$$
x_{n+1}=b x_{n}(\bmod 1),
$$

with initial condition $x_{0}=\theta(\bmod 1)$. The $b$-expansion of a real number $\theta \in[0,1]$ is

$$
\theta=\sum_{j=1}^{\infty} d_{j} b^{-j}
$$

in which the $j$-th digit is defined by $d_{j}:=\left\lfloor b x_{j-1}\right\rfloor$. In Section 3 we suppose the given real number $\theta$ is expressed as

$$
\theta=\sum_{n=1}^{\infty} \varepsilon_{n} b^{-n},
$$

in which $\varepsilon_{n}$ is any sequence of real numbers with $\varepsilon_{n} \rightarrow 0$ as $n \rightarrow \infty$. To this one can associate a perturbed $b$-expansion associated to the perturbed $b$ transformation

$$
y_{n+1}=b y_{n}+\varepsilon_{n+1}(\bmod 1),
$$

starting with an initial condition $y_{0} \in[0,1)$. The recurrence (1-3) is an infinite sequence of maps which change at each iteration. Associated to this recurrence is the perturbed b-expansion

$$
y_{0}+\theta=\sum_{j=1}^{\infty} \tilde{d}_{j} b^{-j}
$$

in which the $j$-th digit is defined by

$$
\tilde{d}_{j}:=\left\lfloor b y_{j-1}+\varepsilon_{j}\right\rfloor .
$$

Choosing the initial condition $y_{0}=0$ gives the perturbed $b$-expansion of $\theta$. The mechanism underlying the approach of Bailey and Crandall is that the the $b$-expansion of $\theta$ and the perturbed $b$-expansion of $\theta$ are strongly correlated in the following sense: The orbit $\left\{y_{n}: n \geq 0\right\}$ of the perturbed $b$-transformation 
with initial condition $y_{0}=0$ asymptotically approaches the orbit $\left\{x_{n}: n \geq 0\right\}$ associated to the $b$-transformation with initial condition $x_{0}=\theta-\lfloor\theta\rfloor$ (Theorem 3.1). In particular, the orbits $\left\{x_{n}: n \geq 0\right\}$ and $\left\{y_{n}: n \geq 0\right\}$ have the same set of limit points, and one is uniformly distributed $(\bmod 1)$ if and only if the other is. This implies that the perturbed $b$-expansion of $\theta$, though different from the $b$-expansion of $\theta$, has similar statistics, in various senses. This connection is quite general, since every real number $\theta$ has representations of the form (1-2).

In Section 4 we consider the particular class of arithmetical constants treated in [Bailey and Crandall 2001], consisting of the countable set of $\theta$ given by an expansion (1-2) with $b \geq 2$ an integer and $\varepsilon_{n}=p(n) / q(n)$, where $p(x), q(x) \in \mathbb{Z}(x)$ and $q(n) \neq$ 0 for all $n \geq 0$. We call such numbers BBP-numbers, and call the associated formula

$$
\theta=\sum_{n=1}^{\infty} \frac{p(n)}{q(n)} b^{-n}
$$

a BBP-expansion to base $b$ of $\theta$. These numbers are named after Bailey, Borwein and Plouffe [Bailey et al. 1997], who demonstrated the usefulness of such representations (when $\operatorname{deg} p(x)<\operatorname{deg} q(x)$ ) in computing base $b$ radix expansions of such numbers. We consider $B P P$-numbers having the additional restriction $\operatorname{deg} p(x)<\operatorname{deg} q(x)$, for this condition is necessary and sufficient for $\varepsilon_{n} \rightarrow 0$ as $n \rightarrow \infty$, so that the results of Section 3 apply. The numbertheoretic character of $B P P$-numbers is that they are special values (at rational points) of functions satisfying a homogeneous linear differential equation with integer polynomial coefficients. We derive the result of Bailey and Crandall that Hypothesis A implies that such $\theta$ either are rational or are normal numbers to base $b$ (Theorem 4.2.) This result makes it of interest to find criteria to determine when BBPnumbers are irrational, which we consider next.

In Section 5 we relate BBP-numbers to the theory of $G$-functions, and characterize the subclass of BBP-numbers which are "special values" of $G$ functions. The subject of $G$-functions has been extensively developed in recent years (see [André 1989; Bombieri 1981; Dwork et al. 1994]) and the special values of such functions can often be proved to be irrational [Bombieri 1981; Chudnovsky 1984; Galochkin 1974]. We observe that BBP-numbers satisfy all but one of the properties required to be a special value of a $G$-function defined over the base field $\mathbb{Q}$. We then show that a BBP-expansion to base $b$ corresponds to a special value of a $G$-series at $z=1 / b$ if and only if the denominator polynomial $q(x)$ (in lowest terms) factors into linear factors over the rationals (Theorem 5.4). We show that if all the roots of $q(x)$ are distinct, then such special values are either rational or transcendental, using Baker's results on linear forms in logarithms, in Theorem 5.5, a result also obtained by Adikhari, Saradha, Shorey and Tijdeman [Adhikari et al. $\geq 2001]$. We summarize other known results about irrationality or transcendence of special values of $G$-functions of the type in Theorem 5.4. It is interesting to observe that every one of the examples given in [Bailey and Crandall 2001] is a special value of a $G$-function. Many other interesting examples of such constants were known earlier; for example, D. H. Lehmer [1975, p. 139] observed that

$$
\sum_{n=0}^{\infty} \frac{1}{(n+1)(2 n+1)(4 n+1)}=\frac{\pi}{3} .
$$

In Section 6 we compare Hypothesis A with a conjecture of Furstenberg in ergodic theory, concerning measures that are ergodic for the joint action of two multiplicatively independent $b$-transformations. Both conjectures have similar conclusions, though there seems to be no direct relation between their hypotheses. Bailey and Crandall have found examples of arithmetical constants which have the property of being BBP-numbers to two multiplicatively independent bases. This suggests that one should look for further conditions under which the two conjectures are more directly related.

In Section 7 we make concluding remarks. We describe an empirical taxonomy of various classes of arithmetical constants, and formulate some alternative classes of arithmetical constants as candidates for inclusion in Hypothesis A.

\section{RADIX EXPANSIONS}

We now consider radix expansions to an integer base $b \geq 2$. Such expansions are obtained by iterating the b-transformation

$$
T_{b}(x)=b x(\bmod 1) .
$$


Given a real number $x_{0} \in[0,1)$, as initial condition, we produce the sequence of remainders

$$
x_{n+1}=b x_{n}(\bmod 1),
$$

with $0 \leq x_{n+1}<1$. That is,

$$
x_{n+1}=b x_{n}-d_{n+1}
$$

where

$$
d_{n+1}=d_{n+1}\left(x_{0}\right)=\left\lfloor b x_{n}\right\rfloor \in\{0,1, \ldots, b-1\}
$$

is called the $n$-th digit of $\theta$. The forward orbit of $x_{0}$ is $O^{+}\left(x_{0}\right)=\left\{x_{n}: n \geq 0\right\}$ and we call $\left\{x_{n}\right\}$ the remainder sequence of the $b$-expansion. Iterating (2-1) $n+1$ times yields

$$
x_{n+1}=b^{n+1} x_{0}-d_{n+1}-b d_{n}-\cdots-b^{n} d_{1} .
$$

Dividing by $b^{n+1}$ yields

$$
x_{0}=\sum_{j=1}^{n} d_{j} b^{-j}-b^{-n-1} x_{n+1} .
$$

Letting $n \rightarrow \infty$ yields the $b$-expansion of $x_{0}$,

$$
x_{0}=\sum_{j=1}^{\infty} d_{j}\left(x_{0}\right) b^{-j}
$$

which is valid for $0 \leq x_{0}<1$. For $\theta \in \mathbb{R}$ we take $x_{0}=\theta-\lfloor\theta\rfloor$ and $d_{0}(\theta)=\lfloor\theta\rfloor \in \mathbb{Z}$, thus obtaining the representation

$$
\theta=d_{0}(\theta)+\sum_{j=1}^{\infty} d_{j}(\theta) b^{-j}
$$

which is called the $b$-expansion of $\theta$. Note that (2-2) gives

$$
x_{n} \equiv b^{n} x_{0}(\bmod 1) \equiv b^{n} \theta(\bmod 1)
$$

in this case.

The following property of $\theta$ concerns the topological dynamics of the $b$-transformation for its iterates.

Definition 2.1. A real number $\theta \in[0,1)$ is digit-dense to base $b$ if, for every $m \geq 1$, every legal digit sequence of digits of length $m$ occurs at least once as consecutive digits in the $b$-expansion

$$
\theta=\sum_{n=1}^{\infty} d_{n}(\theta) \beta^{-n}
$$

The following property of $\theta$ concerns the metric dynamics of the $b$-transformation for its iterates.
Recall that the uniform measure or Lebesgue measure $\mu_{\text {Leb }}$ on $[0,1]$ is the unique absolutely continuous invariant measure for the $b$-transformation $T_{b}$.

Definition 2.2. A real number $\theta \in[0,1)$ is normal to base $b$ if for every $m \geq 1$ every digit sequence $d_{1} d_{2} \cdots d_{m} \in\{0,1, \ldots, d-1\}^{m}$ occurs with limiting frequency $b^{-m}$, as given by the invariant measure $\mu_{\text {Leb }}$.

Recall that

$$
\mu_{\text {Leb }}\left(\left\{x_{0}: d_{1}\left(x_{0}\right) \cdots d_{n}\left(x_{0}\right)=d_{1} d_{2} \cdots d_{m}\right\}\right)=b^{-m} .
$$

It is well known that, for each $b \geq 2$, the set of $\theta \in[0,1]$ that are normal to base $b$ has full Lebesgue measure.

The properties of the digit expansion $\left\{d_{n}(\theta): n \geq 1\right\}$ can be extracted from the remainder sequence $\left\{x_{n}\right\}$. The following result is well known.

Theorem 2.3. Consider an integer base $b \geq 2$ and $a$ real number $\theta \in[0,1]$.

(1) $\theta$ is digit-dense to base $b$ if and only if its remainder sequence $\left\{x_{n}(\theta): n \geq 1\right\}$ to base $b$ is dense in $[0,1]$.

(2) $\theta$ is normal to base $b$ if and only if its remainder sequence $\left\{x_{n}: n \geq 1\right\}$ to base $b$ is uniformly distributed in $[0,1]$.

(3) $\theta$ has an eventually periodic b-expansion if and only if its remainder sequence $\left\{x_{n}: n \geq 1\right\}$ to base $b$ has finitely many limit points. This condition holds if and only if $\left\{x_{n}: n \geq 1\right\}$ eventually enters a periodic orbit of the b-transformation, i.e., $x_{m}=x_{m+p}$ for some $m, p \geq 1$. These equivalent conditions hold if and only $\theta$ is rational.

Proof. (1) The set

$I\left(d_{1} d_{2} \cdots d_{m}\right):=\left\{\theta \in[0,1]: d_{1}(\theta) \cdots d_{m}(\theta)=d_{1} \cdots d_{m}\right\}$ is a half-open interval $\left[a, a+b^{-m}\right)$ of length $b^{-m}$, and the $b^{m}$ intervals partition $[0,1]$. Digit-denseness implies there exists some $x_{k} \in I\left(d_{1} \cdots d_{m}\right)$. This holds for all $m \geq 1$ and generates a dense set of points.

(2) If $\left\{x_{n}: n \geq 1\right\}$ is uniformly distributed $(\bmod 1)$, then the correct frequency of points occurs in each interval $I\left(d_{1} \cdots d_{m}\right)$, and this proves normality of $\theta$. For the converse, one uses the fact that $I\left(d_{1} \cdots d_{m}\right)$ is a basis for the Borel sets in $[0,1)$.

(3) The key point to check is that if the limit set of $\left\{x_{n}: n \geq 1\right\}$ is finite, then this finite set 
forms a single periodic orbit of the $b$-transformation, and some $x_{n}$ lies in this orbit. We omit details; see [Bailey and Crandall 2001, Theorem 2.8].

Remark. Most of the results above generalize to the $\beta$-transformation $T_{\beta}(x)=\beta x(\bmod 1)$ for a fixed real $\beta>1$; these maps were studied by Parry [1960]. Associated to this map is the notion of a $\beta$-expansion for any real number $\theta$, in which the allowed digits are $\{0,1,2, \ldots,\lfloor\beta\rfloor\}$. Not all digit sequences are allowed in $\beta$-expansions, but the set of allowed digit sequences was characterized by Parry; see [Flatto et al. 1994] for other references. One defines a number $\theta$ to be digit-dense to base $\beta$ if every allowable finite digit sequence occurs in its $\beta$-expansion. There is a unique absolutely continuous invariant measure $d \mu$ of total mass one for the $\beta$-transformation, and one defines a number $\theta$ to be normal to base $\beta$ if every finite block of digits occurs in its $\beta$-expansion with the limiting frequency prescribed by this invariant measure. With these conventions, Theorem 2.3 remains valid for a general base $\beta$, except that Theorem 2.3(3) must be taken only as characterizing eventually periodic orbits of the $\beta$-transformation. That is, the final assertion in (3) that $\theta$ is rational must be dropped; it does not hold for general $\beta$. For results relating normality of numbers in different real bases $\beta$, see [Brown et al. 1997].

\section{PERTURBED RADIX EXPANSIONS}

Let $b \geq 2$ be an integer, and let $\left\{\varepsilon_{n}: n \geq 1\right\}$ be an arbitrary sequence of real numbers satisfying

$$
\lim _{n \rightarrow \infty} \varepsilon_{n}=0 \text {. }
$$

Set

$$
\theta=\theta\left(b,\left\{\varepsilon_{n}\right\}\right):=\sum_{n=1}^{\infty} \varepsilon_{n} b^{-n}
$$

We can study the real number $\theta$ using a perturbed $b$-expansion associated to the sequence $\left\{\varepsilon_{n}\right\}$.

The perturbed $b$-transformation on $[0,1)$ is the recurrence

$$
y_{n+1}=b y_{n}+\varepsilon_{n+1} \quad(\bmod 1),
$$

with $0 \leq y_{n+1}<1$ and with given initial condition $y_{0}$. That is,

$$
y_{n+1}=b y_{n}+\varepsilon_{n+1}-\tilde{d}_{n+1},
$$

where, for $n \geq 0$,

$$
\tilde{d}_{n+1}=\left\lfloor b y_{n}+\varepsilon_{n+1}\right\rfloor \in \mathbb{Z}
$$

is the $(n+1)$-st digit of the expansion. The digit sequence $\tilde{d}_{n}=d_{n}\left(y_{0}\right)$ and remainder sequence $\left\{y_{n}\right.$ : $n \geq 1\}$ depend on the initial condition $y_{0}$. Since $\varepsilon_{n} \rightarrow 0$, for all sufficiently large $n$, one has

$$
\tilde{d}_{n} \in\{-1,0,1, \ldots, b-1, b\} .
$$

Now (3-2) iterated $n+1$ times yields

$y_{n+1}=\varepsilon_{n+1}+b \varepsilon_{n}+\cdots+b^{n} \varepsilon_{1}+b^{n+1} y_{0}-\sum_{j=0}^{n} \tilde{d}_{n+1-j} b^{j}$.

Dividing by $b^{n+1}$ yields

$$
\sum_{j=1}^{n+1} \tilde{d}_{j} b^{-j}=\sum_{j=1}^{n+1} \varepsilon_{j} b^{-j}+\left(y_{0}-b^{-n-1} y_{n+1}\right) .
$$

Letting $n \rightarrow \infty$ yields the perturbed b-expansion

$$
y_{0}+\theta=\sum_{j=1}^{\infty} \tilde{d}_{j}\left(y_{0}\right) b^{-j}
$$

valid for $0 \leq y_{0}<1$. We write $y_{n}=y_{n}\left(y_{0}\right)$ for the remainder sequence in (3-2)

The perturbed b-expansion $\left\{d_{n}^{*}(\theta): n \geq 1\right\}$ for $\theta$ given by $(3-1)$ is obtained by choosing the initial condition $y_{0}=0$, i.e., $d_{n}^{*}(\theta):=\tilde{d}_{n}(0)$. We also have the perturbed remainders $\left\{y_{n}^{*}(\theta): n \geq 1\right\}$ given by $y_{n}^{*}(\theta)=y_{n}(0)$.

The main observation of this section is that the remainders of the perturbed $b$-expansion of such $\theta$ are related to the remainders of their $b$-expansion.

Theorem 3.1. Let $b \geq 2$ be an integer and let $\theta:=$ $\sum_{n=1}^{\infty} \varepsilon_{n} b^{-n}$, where $\varepsilon_{n}$ are real numbers with $\varepsilon_{n} \rightarrow 0$ as $n \rightarrow \infty$. Let $\left\{y_{n}^{*}(\theta): n \geq 1\right\}$ denote the associated perturbed remainder sequence of $\theta$, and $\left\{x_{n}(\theta)\right.$ : $n \geq 1\}$ the remainder sequence of its b-expansion. If

$$
t_{n}:=\sum_{j=1}^{\infty} \varepsilon_{n+j} b^{-j}
$$

then

$$
x_{n}(\theta)=y_{n}^{*}(\theta)+t_{n} \quad(\bmod 1) .
$$

The orbits $\left\{x_{n}(\theta): n \geq 1\right\}$ and $\left\{y_{n}^{*}(\theta): n \geq 1\right\}$ asymptotically approach each other on the torus $\mathbb{T}=$ $\mathbb{R} / \mathbb{Z}$ as $n \rightarrow \infty$. 
Proof. Since $y_{0}=0$, formula (3-3) gives

$$
y_{n+1}=\sum_{j=1}^{n+1} b^{n+1-j} \varepsilon_{j} \quad(\bmod 1) .
$$

Now

$$
b^{n} \theta=\sum_{j=1}^{\infty} b^{n-j} \varepsilon_{j}=\sum_{j=1}^{n} b^{n-j} \varepsilon_{j}+t_{n} .
$$

Thus

$$
b^{n} \theta=y_{n}+t_{n} \quad(\bmod 1) .
$$

For the $b$-expansion, $(2-3)$ gives $b_{n} \theta \equiv x_{n}(\bmod 1)$, and combining this with (3-5) yields (3-4).

Since $\varepsilon_{n} \rightarrow 0$ as $n \rightarrow \infty$, we have $t_{n} \rightarrow 0$ as $n \rightarrow \infty$. Thus $\left|x_{n}(\theta)-y_{n}^{*}(\theta)\right| \rightarrow 0$ on $\mathbb{T}$ as $n \rightarrow \infty$. Note that on $\mathbb{T}=\mathbb{R} / \mathbb{Z}$ the points $\varepsilon$ and $1-\varepsilon$ are close.

Lemma 3.2. Let $\left\{x_{n}: n \geq 1\right\}$ and $\left\{y_{n}: n \geq 1\right\}$ be any two sequences in $[0,1]$ with $x_{n}=y_{n}+\delta_{n}(\bmod 1)$ with $\delta_{n} \rightarrow 0$ as $n \rightarrow \infty$.

(1) The sequences $\left\{x_{n}: n \geq 1\right\}$ and $\left\{y_{n}: n \geq 1\right\}$ have the same sets of limit points, provided the endpoints 0 and 1 are identified.

(2) The sequence $\left\{x_{n}: n \geq 1\right\}$ is uniformly distributed $(\bmod 1)$ if and only if $\left\{y_{n}: n \geq 1\right\}$ is uniformly distributed $(\bmod 1)$.

Proof. (1) This is clear since $x_{n_{j}} \rightarrow \psi$ implies $y_{n_{j}} \rightarrow$ $\psi$ and vice-versa, except at the endpoints $\psi=0$ or 1 , which, by convention, we identify as the same point.

(2) This is well known; see [Kuipers and Niederreiter 1974, Theorem 1.2, p. 3].

One can compare the $b$-expansion $\left\{d_{n}(\theta): n \geq 1\right\}$ and the perturbed $b$-expansion $\left\{d_{n}^{*}(\theta): n \geq 1\right\}$ of such $\theta$. We have

$d_{n}(\theta)=\left\lfloor b x_{n-1}\right\rfloor$,

$d_{n}^{*}(\theta)=\left\lfloor b y_{n-1}+\varepsilon_{n}\right\rfloor=\left\lfloor b\left(x_{n-1}-t_{n-1}(\bmod 1)\right)+\varepsilon_{n}\right\rfloor$.

Since $t_{n} \rightarrow 0$ and $\varepsilon_{n} \rightarrow 0$ as $n \rightarrow \infty$, one expects that "most" digit values of the two expansions will agree, in the sense that $d_{n}(\theta)=d_{n}^{*}(\theta)$ for "most" sufficiently large values of $n$. (This is an unproved heuristic statement. It is an open problem to prove that a natural density-one proportion of all $n$ have $d_{n}(\theta)=d_{n}^{*}(\theta)$.) However there is still room for there to be infinitely many $n$ where $d_{n}(\theta) \neq d_{n}^{*}(\theta)$.
We next consider perturbed $b$-expansions having a finite number of limit points, and show that they correspond to rational $\theta$.

Theorem 3.3. Let $b \geq 2$ be an integer and let $\theta=$ $\sum_{n=1}^{\infty} \varepsilon_{n} b^{-n}$ with $\varepsilon_{n}$ a sequence of real numbers with $\varepsilon_{n} \rightarrow 0$ as $n \rightarrow \infty$. The following conditions are equivalent.

(i) $\theta \in \mathbb{Q}$.

(ii) The remainders $\left\{y_{n}^{*}(\theta): n \geq 1\right\}$ of the perturbed $b$-expansion of $\theta$ have finitely many limit points in $[0,1]$.

(iii) The orbit $\left\{y_{n}^{*}(\theta): n \geq 1\right\}$ of the perturbed $b$ transformation asymptotically approaches a periodic orbit

$$
\left\{x_{k}: 0 \leq k \leq p\right\}
$$

of the b-transformation, with $T_{b}\left(x_{k}\right)=x_{k+1}$ and $T_{b}\left(x_{p}\right)=x_{0}$ and for $0 \leq j \leq p-1$. That is,

$y_{n}^{*}(\theta)=x_{j}+\delta_{n}(\bmod 1)$ if $n \equiv j(\bmod p)$ (3-6) with $\delta_{n} \rightarrow 0$ as $n \rightarrow \infty$.

Proof. (i) $\Longrightarrow$ (ii). By Theorem 2.3 if $\theta \in \mathbb{Q}$ the remainders $\left\{x_{n}(\theta): n \geq 1\right\}$ of the $b$-transformation have finitely many limit points. By Theorem 3.1 and Lemma 3.2 we conclude that $\left\{y_{n}^{*}(\theta): n \geq 1\right\}$ has the same set of limit points.

(ii) $\Longrightarrow$ (iii). By Theorem 3.1 and Lemma 3.2 the limit points of $\left\{y_{n}^{*}(\theta): n \geq 1\right\}$ are the same as $\left\{x_{n}(\theta): n \geq 1\right\}$. By Theorem 2.3 such limit points must form a periodic orbit of the $b$-transformation.

(iii) $\Longrightarrow$ (i). The values $\left\{y_{n}^{*}(\theta): n \geq 1\right\}$ have limit points the periodic orbit $\left\{x_{j}: 1 \leq j \leq n\right\}$ of $T_{b}$. By Theorem 2.3, it follows that $\theta \in \mathbb{Q}$.

Remarks. 1. Any real number $\theta$ has some perturbed $b$-expansion that satisfies the hypotheses of Theorem 3.1, so in a sense these expansions are completely general. It follows from Theorem 3.3 that Hypothesis A cannot be valid for all such $\theta$, since there exist irrational $\theta$ that are not normal numbers.

2. The rationality criterion of Theorem 3.3 is not directly testable computationally, unless all $\varepsilon_{n}=0$ for $n \geq n_{0}$; the latter case is essentially the same as that of a $b$-transformation. When infinitely many $\varepsilon_{n}$ are nonzero, then the points $\left\{y_{n}^{*}(\theta): n \geq 1\right\}$ stay outside the periodic orbit for infinitely many values 
of $n$, and the role of the $\left\{\varepsilon_{n}\right\}$ is to compensate for the expanding nature of the map $T(x)=b x(\bmod 1)$ by providing negative feedback to push the iterates closer and closer to the periodic orbit.

(3) Theorem 3.1 does not extend to $\beta$-expansions for noninteger $\beta$. One can consider

$$
\theta=\theta\left(\beta,\left\{\varepsilon_{n}\right\}\right):=\sum_{n=1}^{\infty} \varepsilon_{n} \beta^{-n} .
$$

and define an associated perturbed $\beta$-transformation in the obvious way. However when $\beta$ is not an integer the analogue of Theorem 3.1 fails to hold, since $(3-5)$ is no longer valid. In particular, Theorem 3.1 does not extend to rational $\beta=b / a>1$, with $a>1$.

\section{BBP-NUMBERS AND HYPOTHESIS A}

We consider expansions of the following special form.

Definition 4.1. A BBP-number to base $b$ is a real number $\theta$ with a representation

$$
\theta=\sum_{n=1}^{\infty} \frac{p(n)}{q(n)} b^{-n}
$$

in which $b \geq 2$ is an integer and $p(x), q(x) \in \mathbb{Z}[x]$ are relatively prime polynomials, with $q(n) \neq 0$ for each $n \in \mathbb{Z}_{\geq 0}$. We call (4-1) a BBP-expansion to base $b$.

The name BBP-number refers to Bailey, Borwein and Plouffe, who introduced this class of numbers [Bailey et al. 1997, p. 904], proving that the $d$-th digit of such a number is computable in time at most $O\left(d \log ^{O(1)} d\right)$ using space at most $O\left(\log ^{O(1)} d\right)$. (Here "computing the $d$-th digit" is understood to mean computing an approximation to $b^{d} \theta(\bmod 1)$ that is guaranteed to be within a specified distance to $b^{d} \theta(\bmod 1)$; usually this determines the $d$-th digit, but it may not, near the endpoints of the digit interval.) In other words, computing digits of a BBPnumber is a problem of complexity class $S C^{*}$, a subclass of $S C$ [Johnson 1990, p. 127].

We mainly consider BBP-numbers that satisfy the extra condition

$$
\operatorname{deg} q(x)>\operatorname{deg} p(x) .
$$

This condition guarantees that $\varepsilon_{n}=p(n) / q(n) \rightarrow 0$ as $n \rightarrow \infty$, which makes Theorem 3.1 applicable.
We now formulate two hypotheses, whose conclusions are in terms of topological dynamics and metric dynamics, respectively. The second of these is Hypothesis A of [Bailey and Crandall 2001].

Weak Dichotomy Hypothesis. Let there be given a perturbed b-transformation with $\varepsilon_{n}=p(n) / q(n)$, where $p(x), q(x) \in \mathbb{Z}[x]$ and $\operatorname{deg} q(x)>\operatorname{deg} p(x)$. Then the orbit $\left\{y_{n}: n \geq 1\right\}$ for $\theta\left(b,\left\{\varepsilon_{n}\right\}\right)$ either has finitely many limit points or else is dense in $[0,1]$.

Strong Dichotomy Hypothesis. Let there be given a perturbed b-transformation with $\varepsilon_{n}=p(n) / q(n)$, where $p(x), q(x) \in \mathbb{Z}[x]$ and $\operatorname{deg} q(x)>\operatorname{deg} p(x)$. Then the orbit $\left\{y_{n}: n \geq 1\right\}$ for $\theta\left(b,\left\{\varepsilon_{n}\right\}\right)$ either has finitely many limit points or is uniformly distributed on $[0,1]$. Equivalently, in measure theoretic terms, the measures

$$
\mu_{N}=\frac{1}{N} \sum_{k=1}^{N} \delta_{y_{k}}
$$

converge in the vague topology as $N \rightarrow \infty$ to a limit measure $\mu$, which is an invariant measure for the $b$ transformation, and which is either a measure supported on a finite set or else is Lebesgue measure on $[0,1]$.

The following conditional theorem is a central result of [Bailey and Crandall 2001]:

Theorem 4.2. Let $\theta$ be a BBP-number to base $b$ whose associated BBP expansion satisfies

$$
\operatorname{deg} q(x)>\operatorname{deg} p(x) .
$$

(1) If the Weak Dichotomy Hypothesis is true, $\theta$ is either rational or digit-dense to base $b$.

(2) If the Strong Dichotomy Hypothesis is true, $\theta$ is either rational or a normal number to base $b$.

Proof. The condition $\operatorname{deg} q(x)>\operatorname{deg} p(x)$ guarantees that $\varepsilon_{n}=p(n) / q(n) \rightarrow 0$ as $n \rightarrow \infty$. Thus Theorem 3.1 applies to the BBP-number

$$
\theta=\sum_{n=1}^{\infty} \frac{p(n)}{q(n)} b^{-n}
$$

(1) By the Weak Dichotomy Hypothesis, the limit set of $\left\{y_{n}^{*}(\theta): n \geq 1\right\}$ is dense in $[0,1]$. Therefore Lemma 3.2(1) implies that the $b$-expansion remainders $\left\{x_{n}(\theta): n \geq 1\right\}$ are dense in $[0,1]$. Theorem 2.3(1) then shows that $\theta$ is digit-dense. 
(2) By the Strong Dichotomy Hypothesis, the sequence $\left\{y_{n}^{*}(\theta): n \geq 1\right\}$ is uniformly distributed in $[0,1]$. Therefore Lemma 3.2(2) implies that $\left\{x_{n}(\theta)\right.$ : $n \geq 1\}$ is uniformly distributed in $[0,1]$. Now $\theta$ is normal to base $b$ by Theorem 2.3(2).

Many examples of BBP-numbers satisfying (4-2) where the associated real number $\theta$ is known to be irrational are given in [Bailey et al. 1997; Bailey and Crandall 2001]. For example for various $b$ one can obtain $\pi, \log 2, \zeta(3)$ etc. Bailey and Crandall also observe that $\zeta(5)$ is a BBP-number, to base $b=2^{60}$, but it remains an open problem to decide if $\zeta(5)$ is irrational. All the examples they give of BBPnumbers are actually of a special form: they are "special values" of $G$-functions defined over $\mathbb{Q}$, as we discuss next.

\section{SPECIAL VALUES OF G-FUNCTIONS}

Definition 5.1. A power series

$$
f(z)=\sum_{n=0}^{\infty} a_{n} z^{n}
$$

defines a $G$-series over the base field $\mathbb{Q}$ if the following conditions hold.

(i) Rational coefficients condition. All the $a_{n}$ are rational, so we may write $a_{n}=p_{n} / q_{n}$, with $p_{n}, q_{n} \in$ $\mathbb{Z}$ such that $\left(p_{n}, q_{n}\right)=1$ and $q_{n} \geq 1$.

(ii) Local analyticity condition. The power series $f(z)$ has positive radius of convergence $r_{\infty}$, and for each prime $p$ the $p$-adic function

$$
f_{p}(z):=\sum_{n=0}^{\infty} a_{n} z^{n},
$$

viewed with $a_{n} \in \mathbb{Q} \subseteq \mathbb{Q}_{p}$, has positive radius of convergence $r_{p}$ in $\mathbb{C}_{p}$, the completion of the algebraic closure of $\mathbb{Q}_{p}$.

(iii) Linear differential equation condition. The power series $f(z)$ formally satisfies a homogeneous linear differential equation in $D=d / d z$ with coefficients in the polynomial ring $\mathbb{Q}[z]$.

(iv) Growth condition. There is a constant $C<\infty$ such that $g_{n}:=\operatorname{lcm}\left(q_{1}, q_{2}, \ldots, q_{n}\right)<C^{n}$ for all $n \geq 1$.
There is an extensive theory of $G$-functions; see [Bombieri 1981; André 1989; Dwork et al. 1994]. For the general definition of a $G$-function over an algebraic number field $K$ see [André 1989, p. 14; Dwork et al. 1994]. $G$-functions have an important role in arithmetic algebraic geometry, where it is conjectured that they are exactly the set of solutions over $\overline{\mathbb{Q}}[z]$ of a geometric differential equation over $\overline{\mathbb{Q}}$, as defined in [André 1989, p. 2]. In any case it is known that the (minimal) homogeneous linear differential equation satisfied by a $G$-series is of a very restricted kind: it must have regular singular points, and these must all have rational exponents, by a result of Katz. See [Bombieri 1981, p. 46; Bombieri and Sperber 1982]. (The growth condition (iv) plays a crucial role in obtaining this result.) It follows that a $G$-series analytically continues to a multivalued function on $\mathbb{P}^{1}(\mathbb{C})$ minus a finite number of singular points [Dwork et al. 1994, p. xiv]. We call this multivalued function a $G$-function.

It is known that the set $\mathcal{G}_{K}$ of $G$-series defined over a number field $K$ forms a ring over $K$, under addition and multiplication, which is also closed under the Hadamard product

$$
f \otimes g(z)=\sum_{n=0}^{\infty} a_{n} b_{n} z^{n} ;
$$

see [André 1989, Theorem D, p. 14].

Definition 5.2. A special value of a $G$-function defined over $K$ is a value $f(b)$, where $b \in K$, which is obtained by analytic continuation along some path from 0 to $b$ that avoids singular points.

Siegel [1929] introduced $G$-functions and observed that irrationality results could be proved for their "special values", but did not give any details. Bombieri [1981] developed the theory of $G$-functions and gave explicit irrationality criteria in specific cases (his Theorem 6) for points close to the center of the circle of convergence of the $G$-series, as a by-product of very general results.

It is easy to show that each BBP-number is a special value of a power series on $\mathbb{Q}$ that satisfies conditions (i)-(iii) of a $G$-series. They do not always satisfy the growth condition (iv), however, and in a subsequent result we give necessary and sufficient conditions for the condition (iv) to hold. 
Theorem 5.3. Let $R(x)=p(x) / q(x) \in \mathbb{Q}(x)$ with $p(x), q(x) \in \mathbb{Q}[x]$, where $(p(x), q(x))=1$ and $q(n) \neq$ 0 for all $n \geq 0$. Set

$$
f(z)=\sum_{n=0}^{\infty} \frac{p(n)}{q(n)} z^{n}
$$

and let $f_{p}(z)$ be the $p$-adic power series obtained by interpreting $p(n) / q(n) \in \mathbb{Q} \subseteq \mathbb{Q}_{p}$. Then the power series $f(z)$ satisfies a homogeneous linear differential equation in $d / d z$ with coefficients in $\mathbb{Q}[z]$, and $f(z)$ has positive radius of convergence in $\mathbb{C}$ and $f_{p}(z)$ has a positive radius of convergence in $\mathbb{C}_{p}$ for all primes $p$.

Proof. For the first assertion, let $p(x)=\sum_{j=0}^{l} a_{j} x^{j}$ and $q(x)=\sum_{j=0}^{m} b_{j} x^{j}$. Then the operator

$$
D:=\frac{d^{l+1}}{d z^{l+1}}(1-z)^{l+1}\left(\sum_{j=0}^{m} b_{j}\left(z \frac{d}{d z}\right)^{j}\right) \in \mathbb{Q}\left[z, \frac{d}{d z}\right]
$$

has the property that

$$
D f(z)=0
$$

Indeed one has

$$
q\left(z \frac{d}{d z}\right) f(z)=\sum_{n=0}^{\infty} p(n) z^{n}=\sum_{j=0}^{l} a_{j}^{\prime}\left(\frac{1}{1-z}\right)^{j+1}
$$

where $a_{j}^{\prime}$ are defined by the polynomial identity

$$
\sum_{j=0}^{l} a_{j} x^{j}=\sum_{j=0}^{l} a_{j}^{\prime}\left(\begin{array}{l}
x \\
j
\end{array}\right)
$$

Multiplying this rational function by $(1-z)^{l+1}$ yields a polynomial of degree $l$ in $z$, which is annihilated by $d^{l+1} / d z^{l+1}$, and this verifies $(5-1)$.

For the second assertion, the power series expansion of $f(z)$ clearly has radius of convergence 1 in $\mathbb{C}$. It is easy to establish that the the $p$-adic series $f_{p}(z)$ has a positive radius of convergence on some $p$-adic disk around zero since $|q(n)| \leq c n^{d}$ cannot contain more than $c d \log n$ factors of $p$.

We now give necessary and sufficient conditions for a power series arising from a BBP-number to be a $G$-series.
Theorem 5.4. Let $R(x)=p(x) / q(x) \in \mathbb{Q}(x)$ with $p(x), q(x) \in \mathbb{Q}[x]$ with $(p(x), q(x))=1$ and with $q(n) \neq 0$ for all $n \geq 0$, and set

$$
f(z)=\sum_{n=0}^{\infty} \frac{p(n)}{q(n)} z^{n} .
$$

Then the power series $f(z)$ is a $G$-series (necessarily defined over $\mathbb{Q}$ ) if and only if $q(x)$ factors into linear factors in $\mathbb{Q}[x]$.

Proof. Suppose first that $q(x)$ factors into linear factors over $\mathbb{Q}$, say

$$
q(x)=A \prod_{j=1}^{r} L_{j}(x)
$$

where $L_{j}(x)=l_{j} x+m_{j}$ with $l_{j}, m_{j}$ relatively prime integers. To show $f(z)$ is a $G$-series, by Theorem 5.3 it suffices to we check the growth condition (iv). Now

$$
\begin{aligned}
\operatorname{lcm}\left(q_{1}, q_{2}, \ldots, q_{n}\right) & \leq \operatorname{lcm}(q(1), q(2), \ldots, q(n)) \\
& \leq|A| \prod_{j=1}^{r} \operatorname{lcm}\left(L_{j}(1), \ldots, L_{j}(n)\right)
\end{aligned}
$$

where $L_{j}(n)=l_{j} x+m_{j}$. Now

$$
\begin{aligned}
\log \operatorname{lcm}[1,2, \ldots, m] & =\sum_{\substack{p, j \\
p^{j} \leq m}} \log p \\
& =\sum_{n=1}^{m} \Lambda(n)=m+o(m)
\end{aligned}
$$

by the prime number theorem. This yields

$$
\operatorname{lcm}[1,2, \ldots, m]=e^{m(1+o(1))}
$$

as $m \rightarrow \infty$. This gives a bound

$$
\begin{aligned}
\operatorname{lcm}\left(L_{j}(1), \ldots, L_{j}(n)\right) & \leq \operatorname{lcm}\left(1,2, \ldots,\left|l_{j}\right| n+\left|m_{j}\right|\right) \\
& \leq e^{\left(\left|l_{j}\right| n+\left|m_{j}\right|\right)(1+o(1))} .
\end{aligned}
$$

Substituting this in (5-2) implies condition (iv).

For the opposite direction, we will show that if $q(x)$ does not factor into linear factors over $\mathbb{Q}$ then condition (iv) does not hold. Nagell [1922] showed that if $q(x) \in \mathbb{Z}[x]$ is an irreducible polynomial of degree $d \geq 2$, then there is a positive constant $c(d)$ with the property that for any $\varepsilon>0$ there is a positive constant $C(\varepsilon)$ such that

$$
\operatorname{lcm}(q(1), q(2), \ldots, q(n))>C(\varepsilon) n^{(c(d)-\varepsilon) n}
$$


holds for all $n \geq 1$. One can prove this result with $c(d)=(d-1) / d^{2}$. Such a lower bound applies to any denominator $q(x)$ that does not split into linear factors over $\mathbb{Q}$. To complete the argument one must bound the possible cancellation between the numerators $p(n)$, and denominators $q(n)$. If $(p(x), q(x))=1$ over $\mathbb{Z}[x]$, then

$$
\prod_{j=1}^{n} \operatorname{gcd}(p(j), q(j)) \leq C^{n}
$$

for a finite constant $C=C(p(x), q(x))$. This follows since

$$
\operatorname{gcd}(p(n), q(n)) \leq C
$$

holds for all $n$, for a suitable $C$. To see this, factor $p(x)=\prod\left(x-\alpha_{i}\right)$ and $q(x)=\Pi\left(x-\beta_{j}\right)$, with $\alpha_{i} \neq \beta_{j}$ for all $i, j$. Then, over the number field $K$ spanned by these roots,

$$
\text { ideal } \operatorname{gcd}\left(\left(n-\alpha_{i}\right),\left(n-\beta_{j}\right)\right) \mid\left(\alpha_{i}-\beta_{j}\right) .
$$

Taking a norm from $K / \mathbb{Q}$ of the product of all these ideals gives the desired constant $C$.

Remarks. 1. It is an interesting open question to determine what is the largest value of $c(d)$ allowed in $(5-3)$. One can show that it cannot be larger than $d-1$.

2. There are many more $G$-functions defined over $\mathbb{Q}$ than those given in Theorem 5.4. The set of $G$ functions defined over $\mathbb{Q}$ is closed under multiplication, so that $(\log (1-z))^{2}$ is a $G$-function, but its power series coefficients around $z=0$ are not given by a rational function. Also, for rational $a, b, c$ the Gaussian hypergeometric function

$$
{ }_{2} F_{1}(a, b, c, z)=\sum_{n=0}^{\infty} \frac{(a)_{n}(b)_{n}}{(c)_{n} n !} z^{n},
$$

is a $G$-function which is not of the above kind for "generic" $a, b, c$; see [André 1996].

According to the results of Section 4, the conclusion of Hypothesis A is really a statement about irrational BBP-numbers. A good deal is known about the irrationality or transcendence of the special values of the $G$-series covered in Theorem 5.4, a topic that we now address.
Theorem 5.5. Let $R(x)=p(x) / q(x) \in \mathbb{Q}(x)$ with $p(x), q(x) \in \mathbb{Q}[x]$, where $(p(x), q(x))=1$ and $q(n) \neq$ 0 for all $n \geq 0$. Set

$$
f(z)=\sum_{n=0}^{\infty} \frac{p(n)}{q(n)} z^{n}
$$

If $q(z)$ factors into distinct linear factors over $\mathbb{Q}$, then for each rational $r$ in the open disk of convergence of $q(z)$ around $z=0$ the special value $f(r)$ is either rational or transcendental. Furthermore there is an effective algorithm to decide whether $f(r)$ is rational or transcendental.

Proof. We only sketch the details, since a similar result has been obtained in [Adhikari et al. $\geq 2001$ ]. See also [Tijdeman $\geq 2001$, Theorem 6].

By expanding $R(x)$ in partial fractions, under the hypothesis that $q(x)$ splits in linear factors over $\mathbb{Q}$ one obtains an expansion of the form

$$
R(x)=p_{0}(x)+\sum_{j=1}^{s} \frac{c_{j}}{x-r_{j}}
$$

in which $p_{0}(x) \in \mathbb{Q}[x]$, and each $c_{j}, r_{j} \in \mathbb{Q}$. In fact $r_{j} \notin \mathbb{Z}_{\geq 0}$, so all denominators $q(n)$ are nonzero. Now if $r_{j}=p_{j} / q_{j}$ then one has a decomposition

$$
\sum_{n=0}^{\infty} \frac{1}{n-r_{j}} z^{j}=p_{j}(z)+\sum_{k=1}^{q_{j}} \beta_{j, k} \log \left(1-z \exp \frac{2 \pi i k}{q_{j}}\right)
$$

in which $p_{j}(z)$ is a polynomial with rational coefficients and the $\beta_{j, k}$ are effectively computable algebraic numbers in the field $\mathbb{Q}\left(\exp \left(2 \pi i / q_{j}\right)\right)$. It follows from this that one can express the function $f(z)$ as a finite sum of terms of the form $a_{j} /(1-z)^{j}$ with rational coefficients plus a finite sum of terms of the form $-\beta_{j, k} \log \left(1-\alpha_{j} z\right)$, the $\beta_{j, k}$ and $\alpha_{j}$ being effectively computable algebraic numbers. The nonlogarithmic terms all combine to give a rational function $R_{0}(z)$ with coefficients in $\mathbb{Q}$. Given a rational $r$ with $0<|r|<1$, it follows that $f(r)$ is a finite sum of linear forms in logarithms with algebraic coefficients, evaluated at algebraic points. Using Baker's transcendence result on linear forms in logarithms [1975, Theorem 2.1], $f(r)$ is transcendental if and only if the sum of all the logarithmic terms above is nonzero. There is also an effective decision procedure to tell whether this sum is zero or not. If the logarithmic terms do sum to zero, then 
the remaining rational function terms sum up to the rational number $f(r)=R_{0}(r)$.

The case where $q(x)$ factors into linear factors over $\mathbb{Q}$ but has repeated factors is not covered in the result above. This case includes the polylogarithm $\operatorname{Li}_{k}(z)=\sum_{n=1}^{\infty} z^{n} / n^{k}$ of order $k$, for each $k \geq 2$. Various results are known concerning the irrationality of such numbers. For example, $\mathrm{Li}_{k}(1 / b)$ is irrational for all sufficiently large integers $b$; see [Bombieri 1981]. In fact it is known that the set of numbers $1, \operatorname{Li}_{1}(p / q), \ldots, \operatorname{Li}_{n}(p / q)$, with $\operatorname{Li}_{1}(z)=\log (1-z)$, are linearly independent over the rationals whenever $|p| \geq 1$ and $|q| \geq(4 n)^{n(n-1)}|p|^{n}$, according to [Nikishin 1979]. For polylogarithms one has $\operatorname{Li}_{k}(1)=$ $\zeta(k)$, also on the boundary of the disk of convergence. It is not known whether $\zeta(k)$ is irrational for odd $k \geq 5$, although a very recent result of $\mathrm{T}$. Rivoal [2000] shows that an infinite number of $\zeta(k)$ for odd $k$ must be irrational.

\section{INVARIANT MEASURES AND FURSTENBERG'S CONJECTURE}

It is well known that for single expanding dynamical system, such as the $b$-transformation $T_{b}$, there always exist chaotic orbits exhibiting a wide range of pathology. For example, there exist uncountably many $\theta \in[0,1]$ whose 2 -transformation iterates $\left\{x_{n}\right\}$ satisfy $\frac{1}{25}<x_{n}<\frac{24}{25}$ for all $n \geq 0$; see [Pollington 1979]. One can obtain ergodic invariant measures of $T_{b}$ supported on the closure of suitable orbits, which for example may form Cantor sets of measure zero.

If one considers instead two $b$-transformations, say $T_{b_{1}}$ and $T_{b_{2}}$, with multiplicatively independent values (this means they generate a nonlacunary commutative semigroup $\mathcal{S}=\left\langle T_{b_{1}}, T_{b_{2}}\right\rangle$ ), then the set of ergodic invariant measures for the whole semigroup is apparently of an extremely restricted form. Furstenberg has proposed the following conjecture, suggested as an outgrowth of his work on topological dynamics [Furstenberg 1967, Section IV]. It is explicitly stated in [Margulis 2000, Conjecture 4].

Furstenberg's Conjecture. Let $a, b \geq 2$ be multiplicatively independent integers. The only Borel measures on $[0,1]$ that are simultaneously invariant ergodic measures for

$$
T_{a}(x)=a x(\bmod 1) \quad \text { and } \quad T_{b}(x)=b x(\bmod 1)
$$

are Lebesgue measure and measures supported on finite sets that are periodic orbits of both $T_{a}$ and $T_{b}$.

Various results concerning this conjecture appear in [Rudolph 1990; Parry 1996; Host 1995; Johnson 1992]. In particular, if there is any exceptional invariant measure violating the conjecture, it must have entropy zero with respect to Lebesgue measure.

Furstenberg's conjecture involves some ingredients similar to Hypothesis A, and its conclusion involves a dichotomy similar to that in Hypothesis A. This makes it natural to ask if there is any relation between the two conjectures. At present none is known, in either direction.

One may look for BBP-numbers $\theta \notin \mathbb{Q}$ which have properties similar to that expressed in the hypothesis of Furstenberg's conjecture, i.e., which possess BBP-expansions to two multiplicatively independent bases. It is known that there exist irrational BBP-numbers $\theta=\sum_{n=1}^{\infty} R(n) b^{-n}$ which do possess BBP-expressions to two multiplicatively independent bases. For example, Bailey and Crandall observe that $\theta=\log 2$ has this property, on taking

$$
b=2 \quad \text { and } \quad R(x)=\frac{1}{x}
$$

and

$$
b=3^{2} \quad \text { and } \quad R(x)=\frac{6}{2 x-1}
$$

see [Bailey and Crandall 2001, eqs. (4), (10)]. They also observe that $\theta=\pi^{2}$ has this property, as it possesses BBP-expansions to bases $b=2$ and $b=3^{4}$, the latter one found by Broadhurst [1999, eq. (212), p. 35].

Question. Do all BBP-numbers which are special values of $G$-functions have BBP-expansions in two multiplicatively independent bases?

To make tighter a possible connection between the two conjectures, one can ask for which numbers does the following weaker version of Hypothesis A hold.

Invariant Measure Hypothesis. Every BBP-number to base $b$ has b-transformation iterates $\left\{x_{n}\right\}$ that are asymptotically distributed according to some limiting measure on $[0,1]$.

It would be interesting to find extra hypotheses on a class of arithmetical constants under which a precise connection can be established between Hypothesis A and Furstenberg's conjecture. 


\section{CONCLUDING REMARKS}

Many of the examples of arithmetical constants arise as special values of $G$-functions defined over the rationals, or at least "special values" of functions satisfying linear differential equations with polynomial coefficients in $\mathbb{Q}[x]$. Based on the known results, one may empirically group these constants into three classes, of apparently increasing order of difficulty of establishing irrationality or transcendence results:

1. special values of $G$-functions $f(p / q)$ defined over the rationals, with $p / q$ inside the disk of convergence of the $G$-series;

2. "singular values" $f(1)$ of such a $G$-function, which are values taken at a singular point of the associated (minimal order) linear differential equation, on the boundary of the disk of convergence of a $G$-series, at which the $G$-expansion converges absolutely; and

3. "renormalized singular values", which are the constant terms in an asymptotic expansion of a $G$ function around a singular point.

In this hierarchy, an arithmetical constant may occur as more than one type. For example, $\pi^{2} / 6=$ $\zeta(2)=\mathrm{Li}_{2}(1)$ occurs as a number of type 2 , but it is also realized as a number of type 1 , which falls in the class of constants considered in this paper. It is a nontrivial problem to determine what is the lowest level in the hierarchy a given constant belongs.

Various constants of types 1 and 2 appear in the renormalization of massive Feynman diagrams, see [Broadhurst 1999; Groote et al. 1999], where $\operatorname{Li}_{4}\left(\frac{1}{2}\right)$ is cited as such a constant. Multiple zeta values and polylogarithms give many examples of type 2, see [Borwein et al. 1997; 2001]. Many of the most interesting arithmetical constants naturally arise as constants of type 2 and 3. For examples, the values $\zeta(k)=\mathrm{Li}_{k}(1)$ appear as constants of type 2 , while Euler's constant appears as a type 3 "renormalized" value at $z=1$ of $\operatorname{Li}_{1}(z)$. The problem of showing the linear independence of all odd zeta values $\zeta(2 n+1)$ over the rationals has recently been of great interest from connections with various conjectures in arithmetical algebraic geometry, see [Goncharov 2000]. Many other examples of constants of type 2 and 3 appear in [Lehmer 1975; Flajolet and Salvy 1998].
I am not aware of any irrationality or transcendence results proved for a constant of type 3 .

One can extend the hierarchy above outside the class of $G$-functions. Bombieri observes that the power series

$$
h(z)=\sum_{n=1}^{\infty} \frac{1}{n\left(n^{2}+1\right)} z^{n}
$$

of BBP-type, which is not a $G$-series, has special value at $z=1$ given by

$$
h(1)=\frac{1}{2} \operatorname{Re} \frac{\Gamma^{\prime}(i)}{\Gamma(i)} .
$$

The value $z=1$ lies on the boundary of the disk of convergence of the power series for this function, and corresponds to type (2) above. Another example is

$$
\sum_{n=1}^{\infty} \frac{(-1)^{n}}{n^{2}+1}=\frac{2 \pi}{e^{\pi}-e^{-\pi}}-1
$$

see [Flajolet and Salvy 1998, p. 18], where many other interesting examples are given.

The relevant special values of a rational power series for the approach of Bailey and Crandall to apply are $z=1 / b$ for integer $b \geq 2$, where the disk of convergence of the associated power series has radius 1. One observes that the theory of $G$-functions provides irrationality results for rational values $z=a / b$, without regard for whether $a=1$ or not. This suggests the following question.

Question. Given a rational value $z=a / b$, with $1<$ $|a|<|b|$, is there an associated dynamical system (possibly higher dimensional) for which an analogue of Theorem 3.3 holds, relating the dynamics of one orbit to the $\beta$-expansion of $\theta$, with $\beta=a / b$ ?

At present there seems to be no evidence strongly favoring a particular class of arithmetical constants for which Hypothesis A might be expected to hold. The discussions of Sections 5 and 6 suggest that one might consider the following classes.

1. The largest class is the set of "special values" of power series $f(z)$ defined over $\mathbb{Q}$ at $z=1 / b$, arising from solutions of $D f(z)=0$ for some $D \in$ $\mathcal{W}:=\mathbb{Q}[z, d / d z]$, whose power-series coefficients $a_{n} \rightarrow 0$ as $n \rightarrow \infty$. This class includes all BBPnumbers. 
2. One could restrict to the subclass of special values $z=1 / b$ of $G$-functions defined over the rationals. However we know of no compelling reason to restrict to special values of $G$-functions.

3. The smallest class consists of a class of arithmetical constants which satisfy extra conditions analogous to the hypotheses of Furstenberg's conjecture. These consist of those constants which are BBP-numbers to at least two multiplicatively independent bases. One might add the further restriction that they also be special values of Gfunctions. As noted in Section 6, this class includes $\pi^{2}$ and $\log 2$.

\section{ACKNOWLEDGEMENTS}

The author thanks E. Bombieri for helpful information concerning $G$-functions, and for suggesting the argument establishing (5-4) in Theorem 5.4. He thanks D. H. Bailey for references, and J. A. Reeds and the referee for helpful comments. Work on this paper was done in part during a visit to the Mathematical Sciences Research Institute, Berkeley, September 2000.

\section{REFERENCES}

[Adhikari et al. $\geq 2001$ ] S. Adhikari, N. Saradha, T. N. Shorey, and R. Tijdeman, "Transcendental infinite sums". To appear.

[André 1989] Y. André, G-functions and geometry, Vieweg, Braunschweig, 1989.

[André 1996] Y. André, "G-fonctions et transcendance", J. Reine Angew. Math. 476 (1996), 95-125.

[Bailey and Crandall 2001] D. H. Bailey and R. E. Crandall, "On the random character of fundamental constant expansions", Experiment. Math. 10:2 (2001), 175-190.

[Bailey et al. 1997] D. Bailey, P. Borwein, and S. Plouffe, "On the rapid computation of various polylogarithmic constants", Math. Comp. 66:218 (1997), 903-913.

[Baker 1975] A. Baker, Transcendental number theory, Cambridge University Press, London, 1975.

[Bombieri 1981] E. Bombieri, "On $G$-functions", pp. 1-67 in Recent progress in analytic number theory (Durham, 1979), vol. 2, edited by H. Halberstam and C. Hooley, Academic Press, London, 1981.
[Bombieri and Sperber 1982] E. Bombieri and S. Sperber, "On the $p$-adic analyticity of solutions of linear differential equations", Illinois J. Math. 26:1 (1982), 10-18.

[Borwein et al. 1997] J. M. Borwein, D. M. Bradley, and D. J. Broadhurst, "Evaluations of $k$-fold Euler/Zagier sums: a compendium of results for arbitrary $k$ ", Electron. J. Combin. 4:2 (1997), RP5.

[Borwein et al. 2001] J. M. Borwein, D. M. Bradley, D. J. Broadhurst, and P. Lisoněk, "Special values of multiple polylogarithms", Trans. Amer. Math. Soc. 353:3 (2001), 907-941.

[Broadhurst 1999] D. J. Broadhurst, "Massive 3-loop Feynman diagrams reducible to $S C^{*}$ primitives of algebras of the sixth root of unity", Eur. Phys. J. C Part. Fields 8:2 (1999), 313-333.

[Brown et al. 1997] G. Brown, W. Moran, and A. D. Pollington, "Normality with respect to powers of a base", Duke Math. J. 88:2 (1997), 247-265.

[Chudnovsky 1984] G. V. Chudnovsky, "On applications of Diophantine approximations", Proc. Nat. Acad. Sci. U.S.A. 81:22 (1984), 7261-7265.

[Dwork et al. 1994] B. Dwork, G. Gerotto, and F. J. Sullivan, An introduction to G-functions, Annals of Math. Studies 133, Princeton Univ. Press, Princeton, NJ, 1994.

[Flajolet and Salvy 1998] P. Flajolet and B. Salvy, "Euler sums and contour integral representations", Experiment. Math. 7:1 (1998), 15-35.

[Flatto et al. 1994] L. Flatto, J. C. Lagarias, and B. Poonen, "The zeta function of the beta transformation", Ergodic Theory Dynam. Systems 14:2 (1994), 237-266.

[Furstenberg 1967] H. Furstenberg, "Disjointness in ergodic theory, minimal sets, and a problem in Diophantine approximation", Math. Systems Theory 1 (1967), $1-49$.

[Galochkin 1974] A. I. Galochkin, "Estimates from below of polynomials in the values of analytic functions of a certain class", Mat. Sbornik 95 (1974), 396-417. In Russian; translated in Math. USSR Sbornik 24 (1974), 385-407.

[Goncharov 2000] A. B. Goncharov, "Multiple $\zeta$ values, Galois groups, and geometry of modular varieties", preprint, 2000. See www.arxiv.org/abs/ math.AG/0005069.

[Groote et al. 1999] S. Groote, J. G. Körner, and A. A. Pivovarov, "Transcendental numbers and the topology 
of three-loop bubbles", Phys. Rev. D (3) 60:6 (1999), 061701, 4. See www.arxiv.org/abs/hep-ph/9904304.

[Host 1995] B. Host, "Nombres normaux, entropie, translations", Israel J. Math. 91:1-3 (1995), 419-428.

[Johnson 1990] D. S. Johnson, "A catalog of complexity classes", pp. 67-161 in Handbook of theoretical computer science, Vol. A, edited by J. van Leewen, Elsevier, Amsterdam, 1990.

[Johnson 1992] A. S. A. Johnson, "Measures on the circle invariant under multiplication by a nonlacunary subsemigroup of the integers", Israel J. Math. 77:1-2 (1992), 211-240.

[Kuipers and Niederreiter 1974] L. Kuipers and H. Niederreiter, Uniform distribution of sequences, WileyInterscience, New York, 1974.

[Lehmer 1975] D. H. Lehmer, "Euler constants for arithmetical progressions", Acta Arith. 27 (1975), 125-142.

[Margulis 2000] G. Margulis, "Problems and conjectures in rigidity theory", pp. 161-174 in Mathematics: frontiers and perspectives, edited by V. Arnold et al., Amer. Math. Soc., Providence, RI, 2000.

[Nagell 1922] T. Nagell, "Zur Arithmetik der Polynome", Abh. Math. Sem. Hamb. Univ. 1 (1922), 179-194.

[Nikishin 1979] E. M. Nikishin, "On the irrationality of the values of the functions $F(x, s)$ ", Mat. Sb. 109:3 (1979), 410-417. In Russian; translated in Math. USSR Sbornik 37:3 (1979), 381-388.
[Parry 1960] W. Parry, "On the $\beta$-expansions of real numbers", Acta Math. Acad. Sci. Hungar. 11 (1960), 401-416.

[Parry 1996] W. Parry, "Squaring and cubing the circleRudolph's theorem", pp. 177-183 in Ergodic theory of $\mathbb{Z}^{d}$ actions (Warwick, 1993-1994), edited by M. Pollicott and K. Schmidt, London Math. Soc. Lecture Note Series 228, Cambridge Univ. Press, Cambridge, 1996.

[Pollington 1979] A. D. Pollington, "On the density of sequence $\left\{n_{k} \xi\right\}$ ", Illinois J. Math. 23:4 (1979), 511515.

[Rényi 1957] A. Rényi, "Representations for real numbers and their ergodic properties", Acta Math. Acad. Sci. Hungar 8 (1957), 477-493.

[Rivoal 2000] T. Rivoal, "La fonction zêta de Riemann prend une infinité de valeurs irrationnelles aux entiers impairs", C. R. Acad. Sci. Paris Sér. I Math. 331:4 (2000), 267-270.

[Rudolph 1990] D. J. Rudolph, " $\times 2$ and $\times 3$ invariant measures and entropy", Ergodic Theory Dynam. Systems 10:2 (1990), 395-406.

[Siegel 1929] C. L. Siegel, "Über einige Anwendungen Diophantischer Approximationen", Abh. Preuss. Akad. Wiss. Phys. Math. Kl. no. 1 (1929), 41-69. Reprinted as pp. 209-266 of his Gesammelte Abhandlungen I, Springer, Berlin, 1966.

[Tijdeman $\geq 2001] \quad$ R. Tijdeman, "Some applications of diophantine approximation", in Proc. Millennial Number Theory Conference.

Jeffrey C. Lagarias, AT\&T Labs - Research, Florham Park, NJ 07932-0971, United States (jcl@research.att.com) 